\title{
Using titer and titer normalized to confluence are complementary strategies for obtaining Chinese hamster ovary cell lines with high volumetric productivity of etanercept
}

Pristovšek, Nuša; Hansen, Henning Gram; Sergeeva, Daria; Borth, Nicole; Min Lee, Gyun; Andersen, Mikael Rørdam; Kildegaard, Helene Faustrup

Published in:

Biotechnology Journal

Link to article, DOI:

10.1002/biot.201700216

Publication date:

2018

Document Version

Peer reviewed version

Link back to DTU Orbit

Citation (APA):

Pristovšek, N., Hansen, H. G., Sergeeva, D., Borth, N., Min Lee, G., Andersen, M. R., \& Kildegaard, H. F. (2018). Using titer and titer normalized to confluence are complementary strategies for obtaining Chinese hamster ovary cell lines with high volumetric productivity of etanercept. Biotechnology Journal, 13(3), [1700216]. https://doi.org/10.1002/biot.201700216

\section{General rights}

Copyright and moral rights for the publications made accessible in the public portal are retained by the authors and/or other copyright owners and it is a condition of accessing publications that users recognise and abide by the legal requirements associated with these rights.

- Users may download and print one copy of any publication from the public portal for the purpose of private study or research.

- You may not further distribute the material or use it for any profit-making activity or commercial gain

- You may freely distribute the URL identifying the publication in the public portal 


\section{Research Article}

\section{Using titer and titer normalized to confluence are complementary strategies for obtaining Chinese hamster ovary cell lines with high volumetric productivity of etanercept $t^{\dagger}$}

Nuša Pristovšek ${ }^{1}$, Henning Gram Hansen"1, Daria Sergeevaํ, Nicole Borth², 3, Gyun Min Lee $^{1,4}$, Mikael Rørdam Andersen ${ }^{5}$ and Helene Faustrup Kildegaard ${ }^{1}$

${ }^{1}$ The Novo Nordisk Foundation Center for Biosustainability, Technical University of Denmark, Kemitorvet 220, 2800 Kgs. Lyngby, Denmark

2Department of Biotechnology, University of Natural Resources and Life Sciences, Muthgasse 18, 1190 Vienna, Austria

${ }^{3}$ Austrian Centre of Industrial Biotechnology (ACIB), Muthgasse 11, 1190 Vienna, Austria ${ }^{4}$ Department of Biological Sciences, KAIST, 291 Daehak-ro, Yuseong-gu, Daejeon 305-701, Republic of Korea

5Department of Biotechnology and Biomedicine, Technical University of Denmark, Søltofts Plads, Building 221, 2800 Kgs. Lyngby, Denmark

Correspondence: Helene Faustrup Kildegaard, The Novo Nordisk Foundation Center for Biosustainability, Technical University of Denmark, Kemitorvet 220, 2800 Kgs. Lyngby, Denmark.

E-mail: hef@biosustain.dtu.dk

Henning Gram Hansen, The Novo Nordisk Foundation Center for Biosustainability, Technical University of Denmark, Kemitorvet 220, 2800 Kgs. Lyngby, Denmark E-mail: hgra@biosustain.dtu.dk

${ }^{\dagger}$ This article has been accepted for publication and undergone full peer review but has not been through the copyediting, typesetting, pagination and proofreading process, which may lead to differences between this version and the Version of Record. Please cite this article as doi: [10.1002/biot.201700216].

This article is protected by copyright. All rights reserved

Received: August 4, 2017 / Revised: December 15, 2017 / Accepted: January 16, 2018 


\begin{abstract}
The selection of clonally-derived Chinese hamster ovary (CHO) cell lines with the highest production rate of recombinant glycoproteins remains a big challenge during early stages of cell line development. Different strategies using either product titer or product titer normalized to cell number are being used to assess suspension-adapted clones when grown statically in microtiter plates. However, no reported study so far has performed a direct head-to-head comparison of these two early reporters for predicting clone performance. Therefore, we developed a screening platform for high-throughput analysis of titer and confluence of etanercept-producing clones. We then performed an unbiased comparison of clone ranking based on either titer or titer normalized to confluence (TTC). Using two different suspension cultivation vessels, we demonstrate that titer- or TTC-based ranking gives rise to the selection of clones with similar volumetric productivity in batch cultures. Therefore, a combinatorial titer- and TTC-based ranking is proposed, allowing for selection of distinct clones with both, high integral viable cell density (IVCD) and high specific productivity features, respectively. This contributes to selection of a versatile panel of clones that can be further characterized and from which the final producer clone can be selected that best fits the production requirements.
\end{abstract}

Keywords: $\mathrm{CHO}$ cells, High-throughput, Industrial Biotechnology, Recombinant proteins, Screening, Cell line development

Abbreviations: CLD, Cell line development; FACS, Fluorescence-activated cell sorting; GS, Glutamine synthetase; HDW, Half-deepwell; HT, High-throughput; IVCD, Integral viable cell density; MSX, Methionine sulfoximine; PI, Propidium iodide; $\boldsymbol{q}_{\boldsymbol{p}}$, Specific productivity; TTC, Titer-to-confluence; VCD, Viable cell density 


\section{Introduction}

Chinese hamster ovary ( $\mathrm{CHO}$ ) cells continue to be the cell factory of choice for large-scale biopharmaceutical production [1]. To produce a therapeutic protein with the required quality properties according to FDA and EMA regulations, a stable clonal cell line has to be generated, enabling long-term and reproducible protein expression [2]. Therefore, the classical cell line generation procedure consists of random integration of the transgene into the genome of host cells, followed by selection of transgene containing cells using antibiotics or other transgene selection strategies like methionine sulfoximine (MSX). Subsequently, single-cell cloning and finally, selection of high-producer clones from the bulk of low- and non-producers are performed [3]. Selection of high-producer clones remains one of the major bottlenecks in cell line development (CLD), taking up four to eight months and consuming valuable financial and human resources [4]. Random transgene integration, different transgene copy numbers and the selection pressure encountered during CLD all contribute to genetically heterogeneous populations of cells that require efficient screening processes. Several strategies have been developed in recent years to improve the ease, throughput and performance of the selection processes, carefully reviewed by Priola et al. [5]. These strategies are to a certain degree used in academic labs, but even more so at industrial scale, where highly automated pipelines are employed [6-8].

One of the the main interests during CLD is selecting clones predictive of commercial performance. In order to increase the chances of obtaining clones with desired highproducing performances, the final product yield (maximum titer) is a key parameter to assess in early stages of CLD. High product yield can be achieved via high specific productivity $\left(q_{p}\right)$ and/or high integral viable cell density (IVCD)[9]. Both IVCD and $q_{p}$ depend on extrinsic factors that differ substantially between small-scale and large-scale cultures [10]. However, both parameters also depend on the intrinsic features of the producer clone that must be selected for during the screening process. When clones 
progress from small-scale to large-scale cultures, they usually undergo several assessment stages, which include different growth vessels. Early assessment stages are known to not be very predictive of behaviour in the final processes $[11,12]$. Nonetheless, early predictions represent a critical decision-making step in the selection process of which clones to discard or conversely, which clones with the most promising features to proceed with. Typically, the primary screening and ranking of suspension-adapted clones cultured statically (without shaking) in microtiter plates is based on either the product concentration (titer) [7] or a proxy of $q_{p}[8,11]$. To the best of our knowledge, a side-by-side comparison of how these two early reporters perform in terms of selecting $\mathrm{CHO}$ clones with high volumetric productivity in suspension cultures has not yet been reported.

In order to investigate the performance of titer and proxy of $q_{p}$ as early reporters for volumetric productivity, we generated a polyclonal cell line by transfecting CHO-S cells with a plasmid co-expressing glutamine synthetase (GS) and etanercept and then applied MSXselection. Etanercept is a homodimeric Fc-fusion therapeutic protein that is considered a potentially difficult-to-express protein due to its highly 0-glycosylated regions [13-15]. We then developed a screening platform that combines enrichment of high $q_{p}$ by surface labelling-based fluorescence activated cell sorting (FACS) with high-throughput (HT) and semi-automated confluence analysis of cells cultured statically as well as determination of titer in 96-well microplates. A so-called titer-to-confluence (TTC) ratio is introduced as a proxy for $q_{p}$ for statically cultured cells. Finally, we performed an unbiased evaluation of titer- and TTC-based ranking as early reporters for growth rate as well as for specific and volumetric productivity. We conclude that using titer and TTC as early reporters results in selection of etanercept clones with similar volumetric productivity in batch cultures. 


\section{Materials and methods}

\subsection{Plasmid design and construction}

A plasmid co-expressing GS and etanercept in a pcDNA3.1(+) backbone (PL_GS-etanercept; Fig. S1) was constructed with uracil-specific excision reagent cloning method using flexible assembly sequence tags, uracil-containing primers and Phusion ${ }^{\circledR}$ Hot-Start Flex polymerase (New England Biolabs, Ipswich, MA), as previously described [16]. The GS expression cassette (pSV40-GS-SV40pA) as well as the vector backbone without the zeocin resistance gene expression cassette were directly amplified from a pcDNA3.1/Zeo(+)-GS vector. The etanercept expression cassette was amplified from a pDRIVE5-etanercept expression vector, which had been made by subcloning a DNA fragment with the coding sequence of etanercept (GeneArt, Regensburg, Germany) into the commercial pDRIVE5 vector (InvivoGen, San Diego, CA). The etanercept expression cassette (mCMV-hEF-1a-5'HTLVetanercept-SV40pA) comprises a promoter, consisting of an mCMV enhancer element, a hEF-1a promoter element and a 5' HTLV untranslated region (UTR). The etanercept coding sequence is based on the amino acid sequence with accession number 7783 from the IMGT database [17]. The nucleotide sequence of the etanercept and GS-expression cassettes are provided in Table S1 and S2, respectively. Plasmids used as PCR templates are listed in Table S3. Assembled PCR fragments were transformed into E. coli Mach1 competent cells (Life Technologies, Carlsbad, CA). All constructs were verified with sequencing and purified using NucleoBond Xtra Midi EF (Macherey-Nagel, Düren, Germany) according to manufacturer's instructions.

\subsection{Cell culture, transfection, and generation of an etanercept-expressing polyclonal cell line}

CHO-S suspension cells (Life Technologies) were grown in CD CHO medium (Life Technologies) supplemented with glutamine synthetase expression medium supplement 
(GSEM; Sigma Aldrich, St. Louis, MO) and $2 \mu \mathrm{L} / \mathrm{mL}$ anti-clumping agent (Life Technologies). Cells were maintained in $125 \mathrm{~mL}$ Erlenmeyer flasks (Corning Inc., Acton, MA), incubated at $37^{\circ} \mathrm{C}, 5 \% \mathrm{CO}_{2}$ at $120 \mathrm{rpm}(25 \mathrm{~mm}$ shaking amplitude $)$ and passaged every 2-3 days. In order to establish a stable etanercept-expressing polyclonal cell line, $4.5 \times 10^{6}$ cells were transfected with $5.4 \mu \mathrm{g}$ of plasmid DNA using Amaxa Cell Line Nucleofector Kit V system (Lonza, Basel, Switzerland) and program U-024 on a Nucleofector ${ }^{\circledR} 2 \mathrm{~b}$ device (Lonza), according to the manufacturer's protocol. Cells were then seeded at $4.5 \times 10^{5}$ cells $/ \mathrm{mL}$ in a $125 \mathrm{~mL}$ shake flask and three days after transfection, selection for GS expression was initiated by adding $25 \mu$ M MSX (Sigma Aldrich; Cat. M5379). After 14 days of selection, cells were subjected to an increased selection pressure (50 $\mu \mathrm{M}$ MSX).

\subsection{Surface labelling of etanercept and FACS}

To enrich for cells with high $q_{p}$, immunostaining of etanercept on the plasma membrane (surface labelling) was performed on the etanercept-expressing cell pool by staining with an anti-human IgG Alexa Fluor 488 antibody (Thermo Fischer Scientific; Cat. A-11013) and propidium iodide (PI, Thermo Fischer Scientific) at $4^{\circ} \mathrm{C}$, as previously described [18]. Two gates were used for sorting cells on FACS (BD FACSJazz ${ }^{\mathrm{TM}}$, BD Biosciences, San Jose, CA): 1) A PI-positive/negative gate dividing dead and viable cells, respectively, 2) A gate enriching for high $q_{p}$ which was set at top 5\% highest Alexa Fluor 488 intensity. Only viable cells were sorted and cells were either gated for top 5\% highest Alexa Fluor 488 intensity or not (surface labelled, top 5\%-enriched and surface labelled, non-enriched, respectively). Cells were bulk ( 1 x $10^{5}$ cells/well) and single-cell sorted into flat-bottom Corning 96-well plate (VWR, Radnor, PA; Cat. 734-0954) and flat-bottom Corning 384-well plates (Sigma Aldrich; Cat. CLS3542-50EA), respectively. Bulk-sorted cells were expanded in suspension and transferred to a 6-well plate, where cells were seeded at a density of $3 \times 10^{5}$ cells $/ \mathrm{mL}$ and cultivated under $50 \mu \mathrm{M}$ MSX selection for three days to assess $q_{p}$. Single cells were sorted in 
$30 \mu \mathrm{l}$ of CD CHO medium, supplemented with GSEM, 1.5\% HEPES (Gibco) and 1x AntibioticAntimycotic (Gibco). 15 days after single-cell sorting, the entire volume of sub-confluent clones incubated statically in the absence of MSX was transferred to $200 \mu \mathrm{l}$ of CD CHO medium, supplemented with GSEM and 1x Antibiotic-Antimycotic in flat-bottom 96-well plates using an epMotion ${ }^{\circledR} 5070$ liquid handling workstation (Eppendorf, Hamburg, Germany). Subsequently, the 384-well microplates were visually inspected in order to ensure that the majority of the cells had been successfully transferred.

\subsection{Titer and confluence-based screening of clones}

Following two days of static incubation in 96-well plates, the single-cell sorted cells were re-suspended by pipetting to ensure an even spread of cells on the bottom of the wells. The day after, all clones were sub-confluent and the confluence (the percentage of the well covered by cells) and the number of cells in each well was estimated by image cytometry analysis using the Celígo Imaging Cell Cytometer (Nexcelom Bioscience, MA) with the labelfree, bright field confluence or direct cell counting application, respectively. $100 \mu \mathrm{L}$ of the spent media from the 96-well plates were aspirated and analyzed for etanercept titer as described below. Titer values were divided by confluence in order to compute titer-toconfluence $(\mathrm{TTC})$ ratios $(\mathrm{TTC}=\operatorname{titer}[\mu \mathrm{g} / \mathrm{mL}] /$ confluence[\%]). Then either TTC or titer alone were used to rank all clones. This initial titer- and TTC-based ranking was used throughout the study in order to evaluate the performance of titer and TTC as early reporters for volumetric productivity.

\subsection{Batch cultivation in 96-well plates in suspension}

Selected single-cell derived clones were expanded without MSX selection into pre-sterilized polystyrene 96-square System Duetz half-deepwell (HDW)-plates (CR1496c, Enzyscreen, Haarlem, Netherlands) capped with autoclaved low-evaporation sandwich Duetz covers 
(CR1296a, Enzyscreen). Plates were incubated in a humidified S41i incubator shaker (New Brunswick, Eppendorf, Hamburg, Germany) at the following conditions: $37^{\circ} \mathrm{C}, 5 \% \mathrm{CO}_{2}, 325$ rpm (25 mm shaking amplitude). In order to assess productivity of clones, cells were seeded in a range of $1 \times 10^{5}$ to $1.5 \times 10^{6}$ cells $/ \mathrm{mL}$. Clones below this seeding range were excluded from the analysis. Viable cell density (VCD) and viability were measured daily. In order to make exponential growth possible throughout the experiment, etanercept titers were determined after two days. $q_{p}$ was calculated as described elsewhere [19]. In parallel with the two-day batch culture experiment, a longer-term batch experiment was performed in 96-HDW plates for the same set of clones. Seeding density was in the range of $1 \times 10^{5}$ to 7.5 $\mathrm{x} 10^{5}$ cells $/ \mathrm{mL}$. Clones outside of this seeding range were excluded from the analysis. Final titer of the entire set of clones were measured when $50 \%$ of the clones reached a viability below $65 \%$.

\subsection{Batch cultivation in shake flasks}

The top clones from each ranking (TTC- and titer-based) were further evaluated in duplicates in $125 \mathrm{~mL}$ Corning shaking flasks (Sigma Aldrich) without MSX selection. Cells were seeded at $4 \times 10^{5}$ cells $\mathrm{mL}^{-1}$ in $40 \mathrm{~mL}$ CD CHO medium, supplemented with GSEM and $2 \mu \mathrm{L} / \mathrm{mL}$ anti-clumping agent. Cells were incubated at $37^{\circ} \mathrm{C}, 5 \% \mathrm{CO}_{2}$ at $120 \mathrm{rpm}(25 \mathrm{~mm}$ shaking amplitude). VCD, viability and etanercept titers were measured daily. Cultures were discontinued when viability dropped below $50 \%$.

\subsection{Viability and viable cell density}

When cells were grown in 96-well HDW microplates, viability and VCD were determined by a combined Hoechst and PI stain on the Celígo Imaging cytometer as previously described [20]. Viability and VCD of cells in 6-well plates and 125-mL Erlenmeyer flasks were 
determined on the NucleoCounter NC-200 Cell Counter (Chemometec, Allerod, Denmark using Via1-Cassettes and the 'Viability and Cell Count Assay Method 2 Assay'.

\subsection{Titer measurements}

Etanercept titers were determined in supernatants by bio-layer interferometry using protein A biosensors and an Octet RED96 system (FortéBio, Pall, Menlo Park, CA) as previously described [21], with an increased shaking speed of 1000_rpm. The absolute titers of etanercept were calculated using a calibration curve generated from a dilution series of Enbrel (Pfizer, New York City, NY; Lot R51698) and absolute quantification was validated by Coomassie-stained SDS-PAGE gels as previously described (data not shown) [22].

\subsection{Statistical analyses}

All statistical analyses were performed using GraphPad Prism 7 software (GraphPad Prism Software Inc., CA). To analyze the correlation between two variables, either linear regression or nonlinear regression was used. Nonlinear regression was used when data points were not equally scattered across the entire y range, and nonlinear regression was performed by applying a straight line or a line through origin model with relative weighting. When nonlinear regression was used, outliers were identified and excluded using the ROUT method [23], where maximum false discovery rate (FDR) was set to $1 \%(Q=1 \%)$. For calculating the statistical significance $(\mathrm{p}<0.05)$ between the data points of two groups, different unpaired parametric or nonparametric $t$ tests were performed. Nonparametric Mann-Whitney test was used when data points were not normally distributed according to the D'Agostino-Pearson test. When variances from two populations were not equal according to F test, Welch's correction was used. 


\section{Results and discussion}

In this study, we set out to compare how product titer as well as product titer normalized to confluence (TTC) of suspension-adapted clones in static cultures perform as early reporters for growth rate, specific and volumetric productivity of $\mathrm{CHO}$ cell suspension batch cultures (see Fig. 1 for details on the study design). We hypothesised that the titer-based ranking is biased towards clones with a fast-growing phenotype in static cultures. Since the growth profile changes dramatically when moving clones from static to suspension culturing conditions $[11,12]$, this type of ranking could give rise to poor predictability of the final clone performance in batch cultures. However, by normalizing titer to confluence (TTC-based ranking), high $q_{p}$ clones would be selected instead. Since $q_{p}$ in our experience is generally less prone to variations between growth formats, we hypothesized that TTCbased selection had the potential to not only select clones with high $q_{p}$, but also with high volumetric productivity.

\subsection{Enrichment for etanercept-producing clones with high $q_{p}$ using surface labelling combined with FACS}

After etanercept-producing polyclonal cell line was established during 14 days of MSX selection (Fig. S2), the polyclonal pool was subjected to surface labelling combined with FACS [18], to enrich for clones with high $q_{p}$. A total of 1920 single cells with PI-negative gating (non-enriched cells) and 8448 single cells with double, PI-negative and top 5\% highest anti-IgG fluorescence gating (top 5\%-enriched cells) were single-cell sorted. 15 days after sorting, approximately $10 \%$ of the single-cell sorted cells were recovered $(n=852)$. Sorting for top 5\%-enriched cells gave rise to a 3-fold increase in $q_{p}$ both for bulk- and single-cell sorted cells (Fig. 2a and 2b, respectively). This is consistent with previous reports that have also demonstrated enrichment for clones with high $q_{p}[6,18,24]$. Moreover, the inherent heterogeneity in etanercept expression obtained from random genomic 
integration of plasmids was observed both before and after surface labelling-based enrichment (Fig. 2b). When comparing the staining profile at the time of the sorting with obtained $q_{p}$ values for all surface labelled, single-cell sorted cells (non-enriched and top 5\%enriched), a positive correlation was observed $\left(R^{2}=0.35 ; R^{2}=0.47\right.$ without outliers; Fig. S3). These results show that surface-labelling combined with FACS is capable of enriching for clones with high $q_{p}$ despite an imperfect correlation between staining intensity and $q_{p}$.

\subsection{High-throughput titer and confluence screening of etanercept-producing clones in static culture}

Screening efforts encompassing several growth formats are usually required to isolate a stable cell line with the desired properties from inherently heterogeneous cell pools. To assess $q_{p}$ in the early phase of CLD, we developed a 96-well screening platform where IgG or Fc-fusion protein titer and confluence of suspension-adapted cells are determined in a HT and automation-friendly manner (Fig. S4). These two measurements can be used to calculate the TTC (titer-to-confluence) ratio as a proxy for $q_{p}$. Similarly, Porter et al. [11] used TTC ratio (referred to as 'specific activity') when assessing clones in 96-well microplates. Whereas 'specific activity' relied on visual inspection of confluence, confluence was determined by image cytometry in the present study (Fig. S5). In addition, a clear linear relationship was observed between confluence and cell numbers (Fig. S6), suggesting that confluence is a valid proxy for cell numbers. A total of 852 surviving clones were cultured statically and screened for titer and confluence within a single day. Approximately $80 \%$ of the 852 screened clones had detectable etanercept titers $(n=672)$ and consequently had a positive TTC value assigned. All clones were then ranked twice, either according to their TTC or titer values. This initial titer- and TTC-based ranking was used throughout the study in order to evaluate the performance of titer and TTC as early reporters for volumetric 
productivity (Table S4). Calculated TTC values were spanning from 0.1 to 94.5 (arbitrary units) and measured titer values were ranging from 0.05 to $2.8 \mu \mathrm{g} / \mathrm{mL}$.

\subsection{Characterisation of selected clones in suspension in 96-half-deepwell plates}

To maximize the number of clones being evaluated in suspension, we characterized the selected clones in the previously described System Duetz 96-HDW plates [20,25]. Top 56 and 57 clones (approximately top 6th percentile of the clones) in the TTC and titer category, respectively, and 48 randomly picked top 5\%-enriched clones were selected for further evaluation (Fig. 1). 40 of the 56 TTC-selected clones and 55 of the 57 titer-selected clones survived expansion into 96-HDW plates, whereas 43 of 48 randomly selected clones survived. The difference in survival rate between the TTC- and titer-selected is probably explained by the lower confluence of TTC-selected clones and by the fact that the majority of the non-surviving clones had relatively low confluence (Fig. S7). 16 clones were overlapping between the TTC and titer categories giving rise to 24 and 39 unique TTC- and titer-selected clones, respectively.

In two-day batch cultures in 96-HDW plates, TTC-selected clones on average initially grew slower compared to both titer-selected clones and randomly selected clones (Fig. 3a). However, clones in the TTC category were more frequently seeded at a lower cell density, which correlated with low growth rates (Fig. S8). By comparing only the clones seeded above $4 \times 10^{5}$ cells $/ \mathrm{mL}$, we observed no difference in growth rate between TTC- and titerselected clones (Fig. S9a). Hence, predominantly TTC-selected clones seeded at a lower cell density $\left(<4 \times 10^{5}\right.$ cells $/ \mathrm{mL}$ ) probably experienced a longer lag phase with a possible negative effect on the cell growth rate during the two days of culture. Similarly, TTCselected clones generally had significantly higher $q_{p}$ compared to titer-selected clones (Fig. 3b). However, when comparing the clones with seeding density above $4 \times 10^{5}$ cells $/ \mathrm{mL}$, no difference in $q_{p}$ between the TTC- and titer-selected clones was observed (Fig. S9b). 
Independent of seeding density, the randomly selected clones had significantly lower $q_{p}$ compared to both the TTC and titer categories (Fig. 3b). While a poor correlation was observed between confluence (static cultures) and specific cell growth rate (suspension cultures) (Fig. S10), a clear correlation was observed between TTC and $q_{p}$ (Fig. 3c). Thus, while growth rate clearly responds to changes in culture conditions consistent with previous reports $[11,12]$, the $q_{p}$ of clones seems to be less affected when going from static incubation to suspension culture.

In order to estimate volumetric productivity (maximum titer) in long-term batch suspension cultures in a HT manner, the selected clones were again cultivated in 96-HDW plates. This time, however, all cultures were maintained longer until more than $50 \%$ of the clones had viability below $65 \%$. As expected, randomly selected clones had lower maximum titers compared to both the TTC- and titer-selected clones (Fig. 3d). However, no significant differences in maximum titers were observed between TTC- and titer-selected clones independent of whether the 16 overlapping clones were included or excluded from the analysis (Fig. 3d). In addition, seeding density did not seem to have a clear impact on the maximum titer as opposed to cell growth rate (Fig. S8). Due to the viability-based termination point, viability of clones was different at the end of the experiment. In fact, $47 \%$ and $30 \%$ of the clones had viability above $65 \%$ in the TTC and titer categories, respectively, suggesting that maximum achievable titers were underestimated to a larger degree for the TTC-selected clones compared to the titer-selected clones.

\subsection{Characterisation of highest ranking clones in shake flasks and comparison to 96- half-deepwell data}

To assess the performance of the TTC- and titer-selected clones more thoroughly, the top 12 of the surviving clones in both categories were subjected to a standard batch culture experiment in shake flasks. Four of these clones were overlapping between the two 
categories, giving rise to eight unique clones in both categories. Clone IDs were assigned to each clone in shake flask batch culture and the clone IDs with the corresponding ranks can be seen in Table S4.

Specific growth rates of TTC- and titer-selected clones were not significantly different from day 0 to 2 in shake flasks, whether or not overlapping clones were included in the comparison (Fig. 4a, left panel; growth curves in Fig. S11). Similarly, no difference in growth rates was observed when comparing the same set of top 12 TTC and titer clones in 96-HDW plates (Fig. S12a). Overall, specific growth rates of the top 12 TTC and titer clones were consistent between the 96-HDW microplates and shake flasks except for clones 10 and 11 (Fig. 4a, right panel), which were seeded at a lower density in 96-HDW microplates (Fig. S8). Interestingly, clones 10 and 11 in shake flasks had comparable cell growth rates to the remaining top 12 TTC and titer-selected clones. This is consistent with the previously mentioned notion that these clones potentially had a longer lag phase in the 96-HDW microplates due to lower seeding density. Importantly, we have previously observed similar growth profiles of CHO-S cells between shake flasks and 96-HDW microplates [20]. Thus, the 96-HDW microplate seems to be a suitable microscale vessel for comparison of clones in batch cultures when seeding densities are aligned.

TTC- and titer-selected clones on average achieved similar $q_{p}$ in different stages of batch culture (day 0-2 and day 3-5), whether or not overlapping clones were included in the comparison (Fig. 4b, left panel; growth curves in Fig. S11). Similarly, we observed no difference in $q_{p}$ when comparing the top 12 TTC and top 12 titer clones in 96-HDW plates (Fig. S12b). Comparison of $q_{p}$ between HDW and shake flasks from day 0 to day 2 demonstrated a positive correlation (Fig. 4 b, right panel) $\left(R^{2}=0.58 ; R^{2}=0.71\right.$ without one outlier), suggesting $q_{p}$ was largely unaffected by different seeding densities or formats. However, $q_{p}$ of the clones was decreasing through the time course of the experiments in the present study (Fig. 2 and Fig. 4b, right panel). In our attempt to do an unbiased evaluation 
of TTC- and titer-based selection, MSX selection pressure was not reintroduced after singlecell sorting in order to keep the media unchanged in all cell culture vessels (Fig. 1). The decrease in $q_{p}$ is likely a consequence of no MSX selection pressure after FACS similar to what has been observed for CHO clonal cell lines obtained by dihydrofolate reductase/methotrexate-based selection [26] and/or the presence of post-transcriptional bottlenecks [27]. Thus, it is important to note that the evaluation of the TTC- and titer-based selection is most likely affected by this loss of productivity. Future studies are warranted to investigate whether TTC- or titer-based selection enriches for clones with stable productivity.

Like $q_{p}$, similar average maximum titers were achieved by the TTC- and titer-selected clones (Fig. 4c, left panel). When comparing maximum titers obtained in HDW and shake flasks, the values correlated moderately (Fig. $4 \mathrm{c}$, right panel) $\left(\mathrm{R}^{2}=0.52\right)$. This moderate correlation is likely a combined consequence of difference in seeding density (Fig. S8) and the viabilitybased termination point in the 96-HDW microplates.

Despite not being able to increase the frequency of isolating clones with higher $q_{p}$, the two clones with the highest $q_{p}$ and volumetric productivity in the shake flask experiment were uniquely found by TTC-based selection. These two clones (clone ID: 2 and 11) were ranked as $2^{\text {nd }}$ and $11^{\text {th }}$ highest TTC clone (151 $1^{\text {st }}$ and $557^{\text {th }}$ highest titer clone, respectively) during the initial screening in static culture. As expected, clone 11, having the highest $q_{p}$ and volumetric productivity also had the lowest growth rate in the early phase (day $0-2$ ) of the batch culture $\left(\mu=0.027 \mathrm{~h}^{-1}\right)$ and the lowest accumulated IVCD $(1.8 \mathrm{e} 7$ cells*day/mL). This clone exhibited quite an extraordinary phenotype in comparison to the other highest ranking TTC and titer clones in shake flasks, having a remarkably higher $q_{p}$, especially from day 3 to 5 (Fig. 4b, left panel). Interestingly, the two clones (clone ID: 13 and 17) with highest volumetric productivity in the titer category were likewise uniquely found by titerbased selection and ranked as $1^{\text {st }}$ and $5^{\text {th }}$ highest titer clones $\left(113^{\text {th }}\right.$ and $126^{\text {th }}$ highest TTC 
clone, respectively) during the initial screening of statically cultured clones. The titerselected clone with highest volumetric productivity had one of the highest growth rates in exponential phase $\left(\mu=0.039 \mathrm{~h}^{-1}\right)$ and highest accumulated IVCDs (3.9e7 cells*day/mL) of the entire clone panel. In addition, higher variance in the TTC category was observed for both $q_{p}$ (Fig. 4b, left panel) and volumetric productivity (Fig. 4c, left panel). This suggests that clones in the TTC category are phenotypically more diverse compared to titer-selected clones. Overall, these observations support the notion that high volumetric productivity can be achieved through high IVCD of clones selected by titer-based ranking or through high $q_{p}$ of clones selected by TTC-based ranking.

It has previously been reported that performance in batch cultures does not necessarily predict the performance in final fed-batch cultures, where feed and different media formulation are contributing to major changes in culture conditions, influencing cell growth, specific and volumetric productivity of clones [11,28]. Due to these unpredictable differences in clonal behaviour between batch culture and fed-batch culture, it might be an advantage to have a panel of phenotypically diverse high-producer clones to test in fedbatch cultures. Such a panel seems achievable by employing a dual titer- and TTC-selection strategy. Accordingly, it seems likely that clones fit-for-purpose for already established commercial protein production processes are present in such diverse clone panels, speeding up the process of cell line commercialization. 


\section{Concluding remarks}

A thorough analysis of the heterogeneity in a polyclonal pool was accomplished by HT screening of 852 clones, using selection of clones based on either titer or TTC. Despite poor correlation observed between confluence of statically cultured clones and cell growth rate of clones in suspension, we observed that $q_{p}$ remains largely unaffected. We were able to show that TTC as a proxy for $q_{p}$ is not a precise reporter for high volumetric productivity in suspension consistent with previous reports $[11,12]$. However, TTC-based ranking was used to identify clones with high volumetric productivity, which would not have been found if using titer-based ranking. We therefore suggest TTC-based selection as an addition to titer-based selection, because it increases the possibility of selecting clones in the early phase of CLD with high specific as well as volumetric productivity. Furthermore, the presented screening platform has been developed to isolate etanercept-producing clones and is therefore applicable for other Fc-fusion proteins as well as IgG variants, which currently constitute the majority of biopharmaceuticals on the market [29]. 


\section{Acknowledgement}

The authors thank Nachon Charanyanonda Petersen and Karen Kathrine Brøndum for their assistance with the FACS, Sara Petersen Bjørn for providing one of the plasmids and Stefan Kol for his assistance with the titer measurements. The authors also thank Anne Tolstrup for valuable discussions. This work was supported by the Novo Nordisk Foundation (NNF10CC1016517). N.P., N.B., H.F.K. and M.R.A are receiving funding from the European Union's Horizon 2020 research and innovation programme under the Marie Skłodowska-Curie grant agreement No. 642663.

\section{Conflict of interest}

The authors declare no financial or commercial conflict of interest. 


\section{References}

[1] J. Zhu, Biotechnol. Adv., 2012, 30, 1158.

[2] F.M. Wurm, Nat. Biotechnol., 2004, 22, 1393.

[3] S.M. Noh, M. Sathyamurthy, and G.M. Lee, Curr. Opin. Chem. Eng., 2013, 2, 391.

[4] S.M. Browne and M. Al-Rubeai, Trends Biotechnol., 2007, 25, 425.

[5] J.J. Priola, N. Calzadilla, M. Baumann, N. Borth, C.G. Tate, and M.J. Betenbaugh, Biotechnol. J., 2016, 11, 853.

[6] S. Shi, R.G.G. Condon, L. Deng, J. Saunders, F. Hung, Y.-S. Tsao, and Z. Liu, J. Vis. Exp., 2011, e3010.

[7] K. Lindgren, A. Salmén, M. Lundgren, L. Bylund, Å. Ebler, E. Fäldt, L. Sörvik, C. Fenge, and U. Skoging-Nyberg, Cytotechnology, 2009, 59, 1.

[8] J.J.C. Hou, B.S. Hughes, M. Smede, K.M. Leung, K. Levine, S. Rigby, P.P. Gray, and T.P. Munro, N. Biotechnol., 2014, 31, 214.

[9] J.Y. Kim, Y.G. Kim, and G.M. Lee, Appl. Microbiol. Biotechnol., 2012, 93, 917.

[10] F. Li, N. Vijayasankaran, A. Shen, R. Kiss, and A. Amanullah, MAbs, 2010, 2, 466.

[11] A.J. Porter, A.J. Racher, R. Preziosi, and A.J. Dickson, Biotechnol. Prog., 2010, 26, 1455.

[12] A.J. Porter, A.J. Dickson, and A.J. Racher, Biotechnol. Prog., 2010, 26, 1446.

[13] S. Houel, M. Hilliard, Y.Q. Yu, N. MeLoughlin, S. Millan Martin, P.M. Rudd, J.P. William, and W. Chen, Anal. Chem., 2014, 86, 567.

[14] P.M. O’Callaghan, M.E. Berthelot, R.J. Young, J.W.A. Graham, A.J. Racher, and D. Aldana, Biotechnol. Prog., 2015, 31, 1187.

[15] B. Hassett, E. Singh, E. Mahgoub, J. O’Brien, S.M. Vicik, and B. Fitzpatrick, MAbs, 2017, 862, 0 .

[16] A.M. Lund, H.F. Kildegaard, M.B.K. Petersen, J. Rank, B.G. Hansen, M.R. Andersen, and U.H. Mortensen, PLoS One, 2014, 9, e96693. 
[17] M-P. Lefranc, V. Giudicelli, Q. Kaas, E. Duprat, J. Jabado-Michaloud, D. Scaviner, C. Ginestoux, O. Clément, D. Chaume, Nucleic Acids Res., 2005, 33, D593.

[18] S.C. Brezinsky, G. Chiang, A. Szilvasi, S. Mohan, R. Shapiro, A. MacLean, W. Sisk, and G. Thill, J. Immunol. Methods, 2003, 277, 141.

[19] L.P. Pybus, G. Dean, N.R. West, A. Smith, O. Daramola, R. Field, S.J. Wilkinson, and D.C. James, Biotechnol. Bioeng., 2014, 111, 372.

[20] H.G. Hansen, C.N. Nilsson, A.M. Lund, S. Kol, L.M. Grav, M. Lundqvist, J. Rockberg, G.M. Lee, M.R. Andersen, and H.F. Kildegaard, Sci. Rep., 2016, 5, 18016 .

[21] L.M. Grav, J.S. Lee, S. Gerling, T. B. Kallehauge, A. H. Hansen, S. Kol, G.M. Lee, L. E. Pedersen, and H. F. Kildegaard, Biotechnol. J., 2015, 10, 1446.

[22] H.G. Hansen, H.F. Kildegaard, G.M. Lee, and S. Kol, Biotechnol. J., 2016, 11, 1648 .

[23] H. J. Motulsky and R.E. Brown, BMC Bioinformatics, 2006, 7, .

[24] J. Pichler, S. Galosy, J. Mott, and N. Borth, Biotechnol. Bioeng., 2011, 108, 386.

[25] W.A. Duetz and B. Witholt, Biochem. Eng. J., 2001, 7, 113.

[26] S.J. Kim, N.S. Kim, C.J. Ryu, H.J. Hong, and G.M. Lee, Biotechnol. Bioeng., 1998, 58, 73.

[27] H.G. Hansen, N. Pristovšek, H.F. Kildegaard, and G.M. Lee, Biotechnol. Adv., 2017, 35, 64.

[28] D. Reinhart, L. Damjanovic, C. Kaisermayer, and R. Kunert, Appl. Microbiol. Biotechnol., 2015, 99, 4645.

[29] G. Walsh, Nat. Biotechnol., 2014, 32, 992. 


\section{Figure legends}

Figure 1. Study design: Evaluating surface labelling enrichment and comparing clone performance obtained by TTC- and titer-based selection strategies

An etanercept-producing polyclonal pool was established by nucleofection of CHO-S cells with a plasmid encoding etanercept and glutamine synthetase (GS) on day 1 and methionine sulfoximine (MSX) selection was subsequently applied. The polyclonal pool was then surface labelled and single-cell sorted and bulk-sorted for: a) propidium iodidenegative cells (non-enriched control) and b) PI negative cells in the top five percent of anti-IgG fluorescent intensity (top 5\%-enriched). Bulk-sorted cells were expanded and their specific productivity $\left(q_{p}\right)$ evaluated in a 6-well plate under MSX selection. Following 15 days of static incubation in the absence of MSX, single-cell colonies were transferred from 384-well to 96-well plates. Following three days of static incubation without MSX selection, clones were screened for titer and confluence by bio-layer interferometry and image cytometry, respectively. All clones were ranked according to titer and titer-toconfluence (TTC) ratio. Four groups were selected for further evaluation in suspension in 96-half-deepwell (96-HDW) microplates: 1) randomly picked top 5\%-enriched clones, 2) randomly picked non-enriched clones, 3) top TTC-selected clones and 4) top titerselected clones. Surface labelling enrichment was evaluated by comparing $q_{p}$ between groups 1) and 2). Clone performance (specific cell growth rate $(\mu), q_{p}$ and volumetric productivity) in batch suspension cultures between TTC- and titer-based selection was compared twice without MSX selection. First, between groups 1), 3) and 4) in 96-HDW microplates, and second, between a reduced number of highest ranking clones from group 3) and 4) in shake flasks. The measurements obtained in 96-HDW microplates and shake flask batch cultures were compared. (1) SC, single-cell; D1, Day 1. 
Figure 2. Surface labelling combined with FACS enriches for etanercept-producing clones with higher $q_{p}$

Etanercept-expressing cell pool was stained with an anti-human IgG Alexa Fluor 488 antibody (surface labelling) and cells were bulk and single-cell sorted for top 5\% highest Alexa Fluor 488 fluorescence. (A) Evaluation of specific productivity $\left(q_{p}\right)$ for bulk-sorted non-enriched and top 5\%-enriched cells. Subsequent to FACS sorting, cells were recovered and maintained in methionine sulfoximine (MSX)-containing medium and $q_{p}$ was assessed in two-days batch cultures (day 39-41 after nucleofection) in a 6-well plate in technical triplicates. Statistical significance was calculated using unpaired $t$ test (bars represent mean). (B) Evaluation of $q_{p}$ for single-cell sorted, randomly picked non-enriched $(n=44)$ and randomly picked top 5\%-enriched $(n=43)$ clones. $q_{p}$ was assessed in two-days batch cultures (day 44-46 after nucleofection) without MSX selection in a 96-half-deepwell (96HDW) microplate. Statistical significance was calculated using Mann-Whitney test (bars represent median with interquartile range (IQR)).

\section{Figure 3. TTC- and titer-selected clones achieve higher specific and volumetric productivity than randomly selected clones in 96-half-deepwell plates}

Selected clones were transferred from static culture to suspension in 96-half-deepwell (HDW) microplates. 46 days after nucleofection, specific cell growth rate $(\mu)$, specific productivity $\left(q_{p}\right)$ was assessed in two-days batch cultures in 96-HDW microplates. In parallel, maximum titer was assessed in 7-days batch cultures in 96-HDW microplates. (A) Comparison of specific growth rate in the exponential phase between randomly picked top 5\%-enriched clones $(n=43)$, top titer-to-confluence (TTC) clones $(n=38)$ and top titer clones $(n=54)$. Statistical significance was calculated using Mann-Whitney test (randomly picked top 5\%-enriched clones vs. top TTC clones; randomly picked top 5\%-enriched clones vs. top titer clones) or Welch's $t$ test (top TTC clones vs. top titer clones) (bars represent 
mean \pm interquartile range (IQR)). (B) Comparison of $q_{p}$ in the exponential phase between randomly picked top 5\%-enriched clones, top TTC clones and top titer clones. Statistical significance was calculated using Mann-Whitney test (bars represent median with IQR). (C) Correlation analysis of $q_{p}$ and TTC ratio for all the clones in 96-HDW microplates with positive TTC values $(n=138)$ using nonlinear regression with a straight line model. $\mathrm{R}^{2}$ without and with relative weighing and elimination of outliers is reported. (D) Comparison of maximum titer between randomly picked top 5\%-enriched clones, top TTC clones and top titer clones. Statistical significance was calculated using Mann-Whitney test (bars represent median with IQR). (1) ${ }^{\Delta} p$ value reported for comparison between groups without the clones present in both groups $(n=16)$. (2) Only clones with a seeding density above $1 \times 10^{5}$ cells $/ \mathrm{mL}$ were analyzed. (3) Overlapping clones marked with green color in panel B and D are clones present in 'random clones top 5\%-enriched' that are also present in either 'top TTC clones' and/or 'top titer clones'.

Figure 4. Top TTC- and titer-based clones achieve similar final volumetric productivity in shake flask batch cultures

Top 12 TTC- and titer clones were transferred from 96-half-deepwell (HDW) microplates to shake flasks. 66-74 days after nucleofection, specific cell growth rate $(\mu)$, specific productivity $\left(q_{p}\right)$ and maximum titer were assessed in batch cultures. Clone performance was compared between 96-HDW microplates and shake flask batch cultures. (A, left panel) Comparison of specific growth rate in the exponential phase of shake flask batch cultures between top titer-to-confluence (TTC) clones $(n=12)$ and top titer clones $(n=12)$. Statistical significance was calculated using unpaired $t$ test (bars represent mean \pm SD). (A, right panel) Correlation analysis of specific growth rates of clones from day 0 to 2 between 96half-deepwell (96-HDW) microplates and shake flask batch cultures ( $n=18)$, using regression through the origin model. (B, left panel) Comparison of $q_{p}$ from day 0-2 and day 
3-5 of shake flask batch cultures between top TTC clones and top titer clones. Statistical significance was calculated using Welch's $t$ test (day 0-2) and Mann-Whitney test (day 3-5) (bars represent median \pm interquartile range (IQR)). (B, right panel) Correlation analysis of specific productivity $\left(q_{p}\right)$ of clones from day 0 to 2 between 96-HDW microplates and shake flask batch cultures, using regression through the origin model. $\mathrm{R}^{2}$ without and with relative weighing and elimination of an outlier is reported. (C, left panel) Comparison of maximum titer in shake flask batch cultures between top TTC clones and top titer clones. Statistical significance was calculated using unpaired $t$ test (bars represent mean \pm SD). (C, right panel) Correlation analysis of maximum titer of clones between 96-HDW microplates and shake flask batch cultures, using regression through the origin model. $\mathrm{R}^{2}$ with relative weighing is reported.

(1) ${ }^{p}$ value for comparison between groups without the clones present in both groups $(n=4)$. (2) Correlation analysis was only performed for clones with seeding density $>1 \times 10^{5}$ cells $/ \mathrm{mL}$ in 96HDW microplates. (3) IDs ranging from 1-20 were assigned to clones in shake flask batch cultures and the corresponding titer- and TTC-based ranks can be seen in Table S4. 


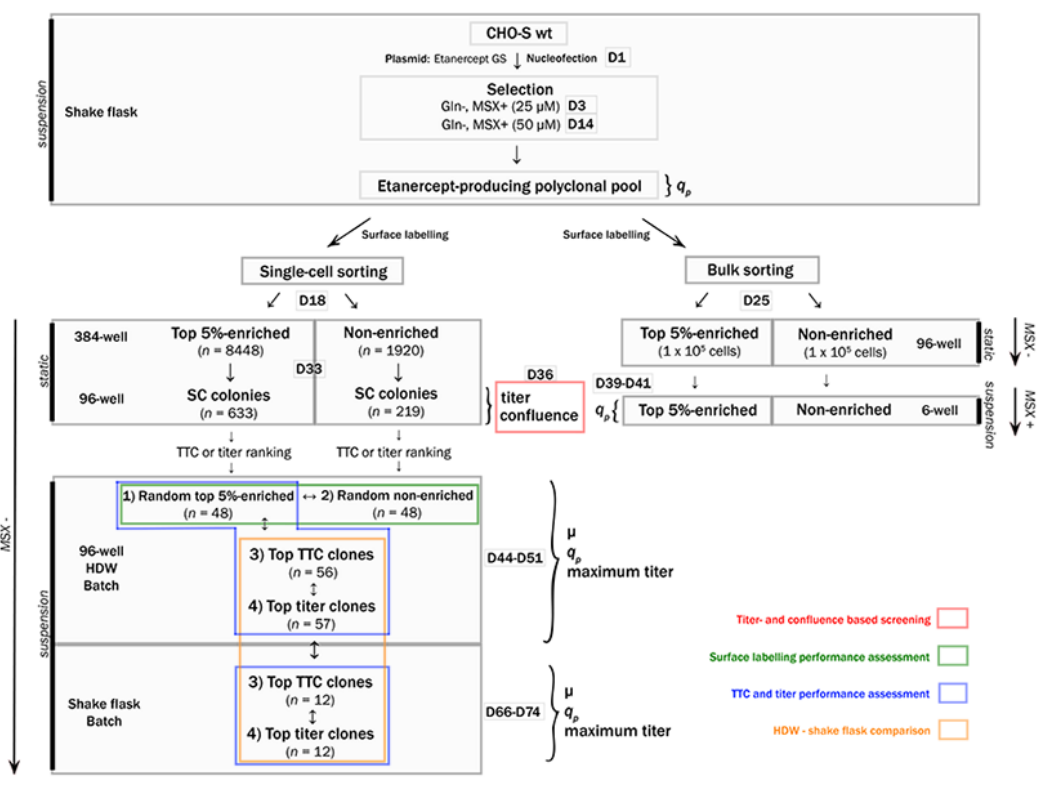

Figure 1 
A

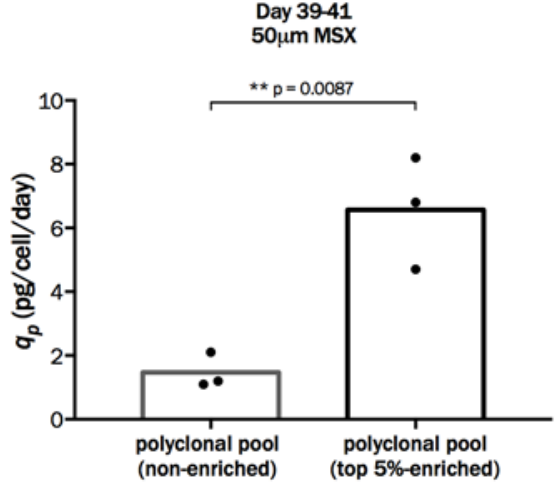

B

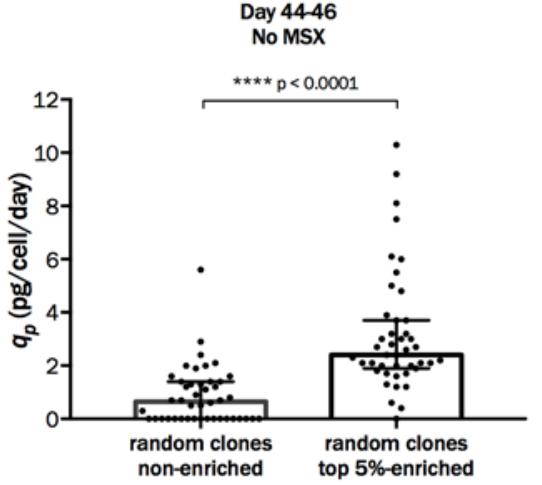

Figure 2 
A
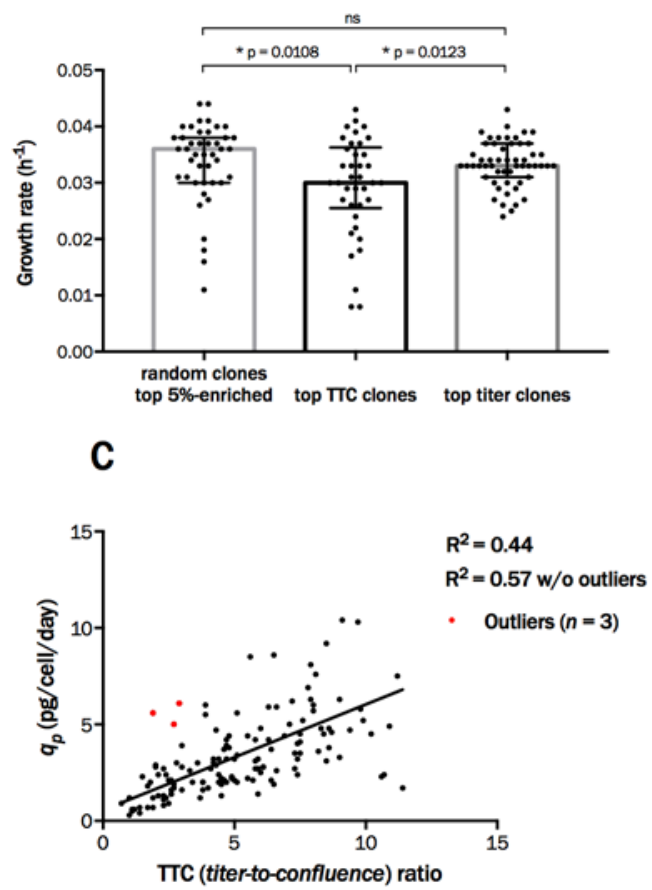

B
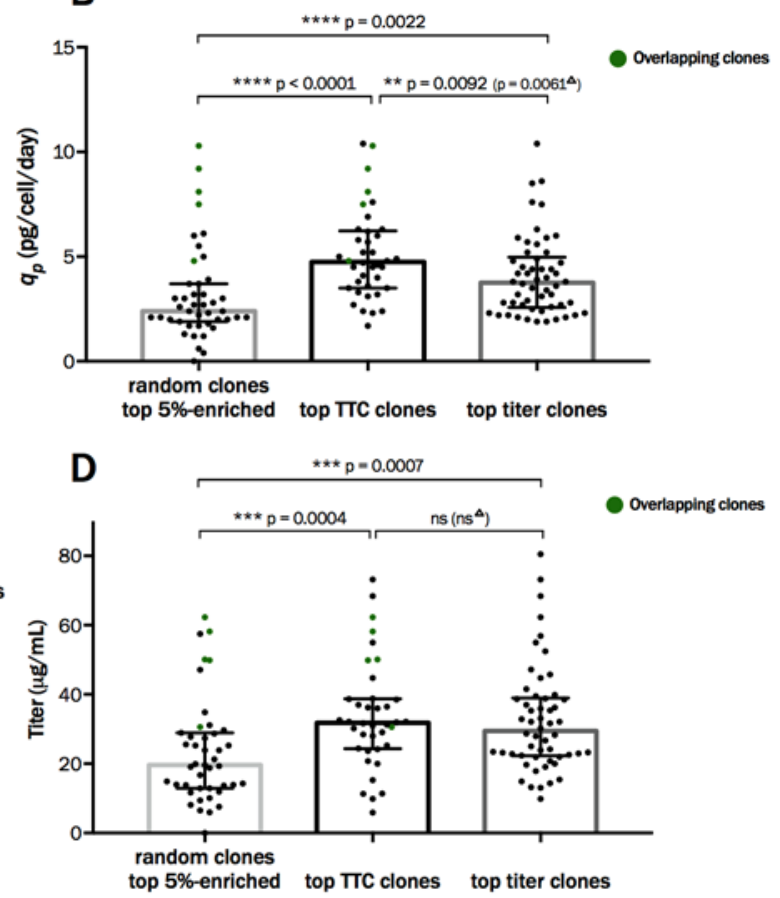

\section{Figure 3}



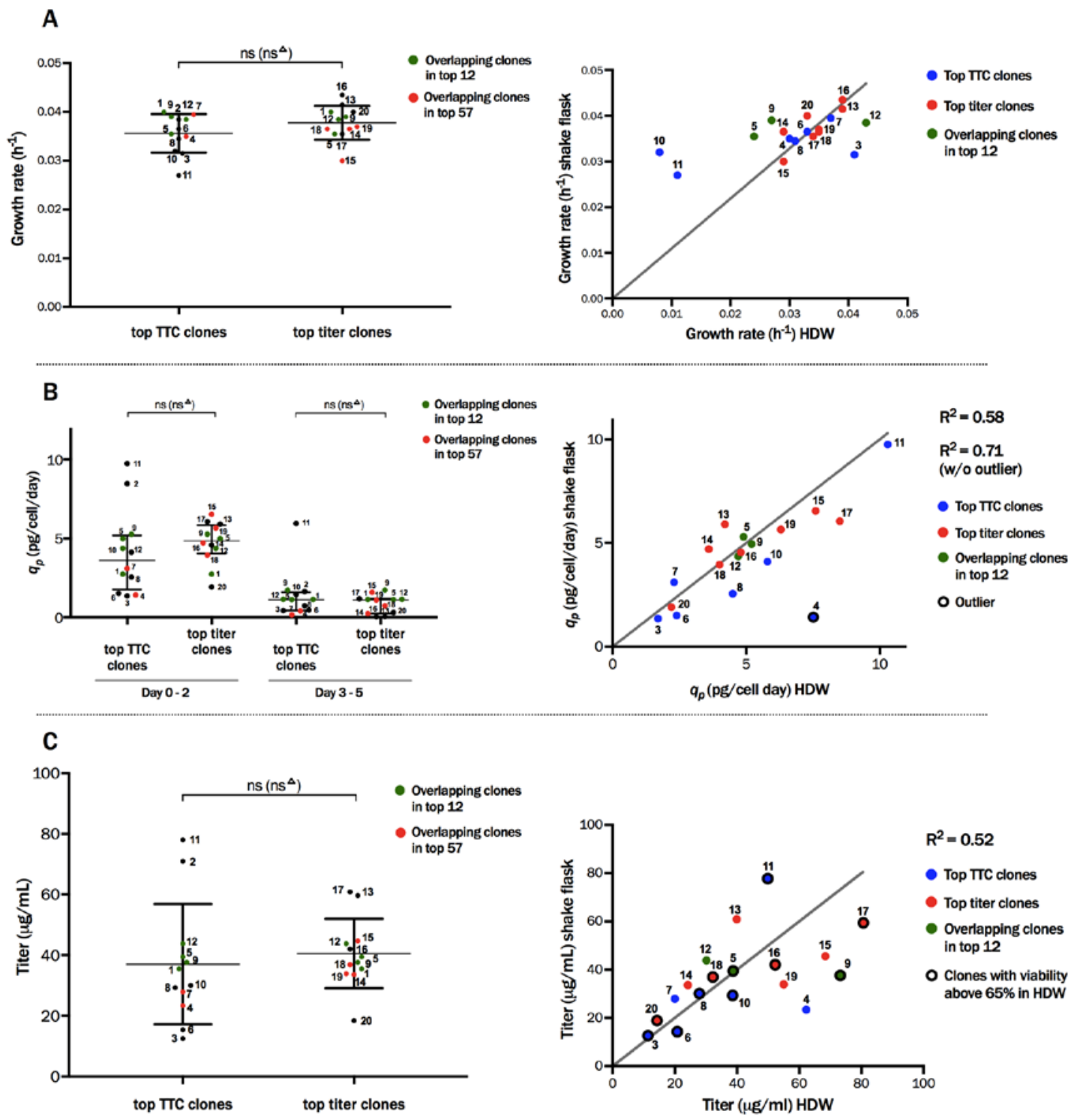

Figure 4 